\title{
The modulational instability in deep water under the action of wind and dissipation
}

\author{
C. KHARIF ${ }^{1} \dagger$, R. A. KRAENKEL $L^{2}$, M. A. MANNA \\ AND R. THOMA ${ }^{1}$ \\ ${ }^{1}$ Institut de Recherche sur les Phénomènes Hors Équilibre, 49, rue F. Joliot-Curie, BP 146, \\ 13384 Marseille CEDEX 13, France \\ ${ }^{2}$ Instituto de Fisica Teorica, UNESP, R. Pamplona 145, 01405-900 São Paulo, Brazil \\ ${ }^{3}$ Laboratoire de Physique Théorique et Astroparticules, CNRS-UMR 5207, Université Montpellier II, \\ Place Eugène Bataillon, 34095 Montpellier CEDEX 05, France
}

(Received 5 February 2010; revised 11 August 2010; accepted 11 August 2010;

first published online 1 November 2010)

The modulational instability of gravity wave trains on the surface of water acted upon by wind and under influence of viscosity is considered. The wind regime is that of validity of Miles' theory and the viscosity is small. By using a perturbed nonlinear Schrödinger equation describing the evolution of a narrow-banded wavepacket under the action of wind and dissipation, the modulational instability of the wave group is shown to depend on both the frequency (or wavenumber) of the carrier wave and the strength of the friction velocity (or the wind speed). For fixed values of the watersurface roughness, the marginal curves separating stable states from unstable states are given. It is found in the low-frequency regime that stronger wind velocities are needed to sustain the modulational instability than for high-frequency water waves. In other words, the critical frequency decreases as the carrier wave age increases. Furthermore, it is shown for a given carrier frequency that a larger friction velocity is needed to sustain modulational instability when the roughness length is increased.

Key words: air/sea interactions, surface gravity waves, wind-wave interactions

\section{Introduction}

Since Stokes (1847), it is well known that the potential water wave problem admits as solutions uniform wave trains of two-dimensional progressive waves. The stability of the Stokes' wave solution started with the work by Lighthill (1965) who provided a geometric condition for wave instability. Later on, Benjamin \& Feir (1967) showed analytically that Stokes waves of moderate amplitude are unstable to small long-wave perturbations travelling in the same direction. This instability is called the Benjamin-Feir instability (or modulational instability). Whitham (1974) derived the same result independently by using an averaged Lagrangian approach, which is explained in his book. At the same time, Zakharov (1968), using a Hamiltonian formulation of the water wave problem obtained the same instability result and derived the nonlinear Schrödinger equation (NLS equation). The evolution of a two-dimensional nonlinear wave train on deep water, in the absence of dissipative effects, exhibits the Fermi-Pasta-Ulam recurrence phenomenon. This phenomenon is characterized by a series of modulation-demodulation cycles in which initially 
nearly uniform wave trains become modulated and then demodulated until they are again nearly uniform. Modulation is caused by the growth of the two dominant sidebands of the Benjamin-Feir instability at the expense of the carrier. During the demodulation, the energy returns to the components of the original wave train. Recently, within the framework of the NLS equation, Segur et al. (2005a) revisited the Benjamin-Feir instability when dissipation is taken into account. The latter authors showed that for waves with narrow bandwidth and moderate amplitude, any amount of dissipation stabilizes the modulational instability. In the wavenumber space, the region of instability shrinks as time increases. This means that any initially unstable mode of perturbation does not grow for ever. Damping can stop the growth of the sidebands before nonlinear interactions become important. Hence, when the perturbations are small initially, they cannot grow large enough for nonlinear resonant interaction between the carrier and the sidebands to become important. The amplitude of the sidebands can grow for a while and then oscillate in time. Segur et al. (2005a) have confirmed their theoretical predictions by laboratory experiments for waves of small to moderate amplitude. Later, Wu, Liu \& Yue (2006) developed fully nonlinear numerical simulations which agreed with the theory and experiments of Segur et al. $(2005 a)$.

Within the framework of random waves, there exists a stochastic counterpart of the modulational instability discussed above. The stability of nearly Gaussian and narrow-banded water wave trains was investigated by Alber \& Saffman (1978), Alber (1978) and Crawford, Saffman \& Yuen (1980). They found that the modulational instability on deep water occurs, provided that the relative spectral width is less than twice the average steepness. Hence, the effect of increasing randomness is to restrict the instability criterion, to delay the onset of modulational instability, and to reduce the amplification rate of the modulation.

From the previous studies we could conclude that dissipation and randomness may prevent the development of the Benjamin-Feir instability (or modulational instability). These two effects question the occurrence of modulational instability of water wave trains. Segur, Henderson \& Hammack (2005b) speculated about the effect of dissipation on the early development of rogue waves and asked the question: can the Benjamin-Feir instability spawn a rogue wave? Since damping affects the modulational instability of waves in deep water, they assumed that it might affect the early development of rogue waves. Nevertheless, the latter study did not include wind effect. What is the role of wind upon modulational instability when dissipative effects are considered? Waseda \& Tulin (1999) showed experimentally that the wind does not suppress the Benjamin-Feir instability even if the naturally (unseeded experiments) developed initial sideband energy is reduced. This finding contrasts with that of Bliven, Huang \& Long (1986) who conducted unseeded experiments and found that sideband growth was reduced in the presence of wind.

The present paper is aimed at reporting on the behaviour of Benjamin-Feir instability when dissipation and wind input are both taken into account. Following Miles (1957), but within the framework of modulated wave trains, we assume the atmospheric pressure at the interface due to wind and the water wave slope to be in phase. The effect is to produce an exponential growth of the wave amplitude. We remind the reader that Miles (1957) studied a linear and uniform monochromatic wave train, whereas we are considering a weakly nonlinear modulated wave train.

In the presence of dissipation it is found that carrier waves of given frequency (or wavenumber) may suffer modulational instability when the friction velocity is larger than a threshold value. Conversely, for a given friction velocity it is found that only 
carrier waves whose frequency (or wavenumber) is less than a threshold value are unstable. Otherwise dissipation prevents instability developing over time.

In $\S 2$, the governing equations and the wave amplification theory of Miles (1957) are briefly presented. In $\S 3$, the NLS equation is introduced when dissipation and wind forcing are considered and the competition between wave amplification by the wind and stabilization due to dissipation is considered. The linear stability analysis of the Stokes-like wave is developed in $\S 4$. The final discussion is found in $\S 5$.

\section{Surface waves under the action of wind and dissipation}

We will consider waves on the surface of a fluid whose viscosity is small as given by Lamb (1993, article no. 349). If $g$ is the acceleration due to gravity, $k$ is the wavenumber of the surface perturbation and $v$ is the viscosity, we define a nondimensional number $\mathscr{L}(k)=v / \sqrt{g / k^{3}}$, and we say that a fluid is of small viscosity if

$$
\mathscr{L}(k) \ll 1 .
$$

For the free surface problem in water, viscous effects are generally weak producing a thin rotational layer adjacent to the potential flow. The thickness of this rotational boundary layer is $O(\sqrt{v / k c})$, where $c$ is the phase velocity. In this context, it was shown by Dias, Dyachenko \& Zakharov (2008) that the equations governing the fluid's motion can be formulated with the help of potential theory. The correction due to viscosity they derived within the framework of the linearized equations was heuristically generalized to the nonlinear equations. Using a similar approach, Lundgren (1989) derived linear versions of the modified boundary equations (2.4) and (2.5). Note that another variant of the introduction of viscous effects within the framework of potential theory can be found in the paper by Skandrani, Kharif \& Poitevin (1996).

The fluid layer is limited above by the water surface described by $z=\eta(x, t)$. We will consider the case of infinite depth. Under these hypotheses, the Laplace equation, the bottom boundary condition and the kinematic condition are

$$
\begin{aligned}
\phi_{x x}+\phi_{z z}=0 & \text { for } \quad-\infty \leqslant z \leqslant \eta(x, t), \\
\nabla \phi \rightarrow 0 & \text { for } \quad z \rightarrow-\infty, \\
\eta_{t}+\phi_{x} \eta_{x}-\phi_{z}-2 v \eta_{x x}=0 & \text { for } \quad z=\eta(x, t) .
\end{aligned}
$$

The dynamic boundary condition is modified by wind effect too, and has the form

$$
\phi_{t}+\frac{1}{2}\left[\left(\phi_{x}\right)^{2}+\left(\phi_{z}\right)^{2}\right]+g \eta=-\frac{1}{\rho} P_{a}-2 v \phi_{z z} \quad \text { for } \quad z=\eta(x, t),
$$

where $\rho$ is the fluid's density and $P_{a}$ is the excess pressure at the free surface. As proposed by Dias et al. (2008), (2.4) and (2.5) are simply heuristic nonlinear generalizations of their linear versions when dissipation is introduced.

In this paper we consider water wave trains in the open ocean far from any solid boundaries. Note that in the case of surface waves generated in wave tanks damping due to viscous dissipation at the lateral solid boundaries must be introduced (see Miles 1967).

The dependence of the fluctuating pressure, $P_{a}$, on $\eta$ is what defines the wind-wave interactions. Within the framework of linearized equations, Miles (1957) assumed that the surface elevation and aerodynamic pressure are $\eta(x, t)=a \mathrm{e}^{\mathrm{i} k(x-c t)}$ and $P_{a}=(\alpha+$ i $\beta) \rho_{a} U_{1}^{2} k \eta$, respectively, where $a$ denotes the amplitude, $k$ is the wavenumber, $c$ is 
the phase velocity, $\alpha$ and $\beta$ are two coefficients depending on both $k$ and $c, \rho_{a}$ is the density of air and $U_{1}$ is a characteristic velocity related to the friction velocity, $u_{*}$, of wind over the water waves. In the expression of $P_{a}$, there is a component in phase and a component in quadrature with the water elevation. For an energy flux to occur from the wind to the water waves, there must be a phase shift between the fluctuating pressure and the interface. Hence, the transfer of energy is only due to the component in quadrature with the water surface or in other words in phase with the slope. To simplify the problem, we consider only the pressure component in phase with the slope on the interface

$$
P_{a}(x, t)=\rho_{a} \beta U_{1}^{2} \eta_{x}(x, t) .
$$

For a logarithmic velocity profile in the turbulent boundary layer over the wave, we have $U_{1}=u_{*} / \kappa$, where $\kappa$ is the von Kármán constant. Hence,

$$
P_{a}(x, t)=\mathscr{W} \eta_{x}(x, t),
$$

where $\mathscr{W}=\rho_{a}\left(\beta / \kappa^{2}\right) u_{*}^{2}$.

The rate of growth of the wave energy is $s \beta \omega\left(u_{*} / c\right)^{2} / \kappa^{2}$, where $s=\rho_{a} / \rho$ is the air-water density ratio and $\omega=k c$ the frequency.

Miles (1957) developed an alternative derivation of the rate of growth of the wave energy based on a linear stability analysis of the parallel shear flows. The transfer of energy from a shear flow $U(z)$ to a surface wave of wavenumber, $k$, and phase velocity, $c$, is associated with a singularity at the critical layer $z=z_{c}$ at which $U\left(z=z_{c}\right)=c$

$$
\frac{\partial \bar{E}}{\partial t}=-\rho_{a} c \pi\left(\frac{\mathrm{d}^{2} U}{\mathrm{~d} z^{2}}\left(z_{c}\right) / k\left|\frac{\mathrm{d} U}{\mathrm{~d} z}\left(z_{c}\right)\right|\right) \overline{w^{2}}\left(z_{c}\right),
$$

where $\bar{E}$ is the mean surface wave energy and $\overline{w^{2}}\left(z_{c}\right)$ is the mean-square value of the wave-induced vertical velocity at $z=z_{c}$. The overbar denotes an average over $x$. The vertical velocity, $w$, is calculated from a Sturm-Liouville differential equation (or the Rayleigh equation). The Rayleigh equation can be solved numerically once $U(z)$, $k$ and $c$ are known. Nevertheless, the presence of a singularity at the critical height, $z_{c}$, complicates the resolution. Conte \& Miles (1959) developed a numerical method to treat this singularity and solve the Rayleigh equation.

Writing $\partial E / \partial t=\gamma E$, we introduce the Miles coefficient $\beta$ such as

$$
\gamma=\frac{\rho_{a}}{\rho} k c \beta\left(\frac{U_{1}}{c}\right)^{2}=s \omega \beta\left(\frac{U_{1}}{c}\right)^{2},
$$

where $\gamma$ is the rate of growth of the wave energy. Following Miles (1996), the coefficient $\beta$ is given by the following expression:

$$
\beta=-\frac{\pi}{k} \frac{\left(\mathrm{d}^{2} U / \mathrm{d} z^{2}\right)\left(z_{c}\right)}{\left|(\mathrm{d} U / \mathrm{d} z)\left(z_{c}\right)\right|} \frac{\overline{w^{2}\left(z_{c}\right)}}{\left.U_{1}^{2} \overline{(\partial \eta / \partial x}\right)^{2}},
$$

where $z=\eta(x)$ is the equation of the surface wave profile.

For a logarithmic profile of the atmospheric shear flow, the rate of growth $\gamma$ is

$$
\gamma=\frac{\rho_{a}}{\rho} k c \beta\left(\frac{u_{*}}{c}\right)^{2} / \kappa^{2}=\frac{s}{\kappa^{2}} \omega \beta\left(\frac{u_{*}}{c}\right)^{2} .
$$

Note that in the classical theory of Miles, the interaction between the wave-induced motion in the air flow and the turbulence is ignored. The turbulence is introduced only to sustain a logarithmic wind profile. 


\section{The perturbed NLS equation: amplification versus depletion}

In this section, we consider both effects of dissipation and wind amplification on the Benjamin-Feir instability. In the absence of viscosity and wind action, the Benjamin-Feir instability may be investigated via an asymptotic expansion leading to the well-known NLS equation. Our aim is to extend the works of Segur et al. (2005a) and Leblanc (2007) who investigated this problem by considering damping and wind effects separately. The derivation of the damped and forced NLS equation does not present any conceptual difficulty. Hence, the expression of the perturbed NLS equation can be stated as

$$
\mathrm{i}\left(\psi_{t}+V \psi_{x}\right)-\frac{\Omega_{0}}{8 k_{0}^{2}} \psi_{x x}-2 \Omega_{0} k_{0}^{2}|\psi|^{2} \psi-\mathrm{i} \frac{\mathscr{W} \Omega_{0} k_{0}}{2 g \rho} \psi=-2 \mathrm{i} \nu k_{0}^{2} \psi,
$$

where $k_{0}$ and $\Omega_{0}$ are the wavenumber and frequency of the carrier wave, respectively, satisfying the linear dispersion relation $\Omega_{0}^{2}=g k_{0}$ and $V=\Omega_{0} / 2 k_{0}$ is the group velocity of the carrier wave. Equation (3.1) describes the spatial and temporal evolution of the envelope, $\psi$, of the surface elevation, $\eta$, of weakly nonlinear and dispersive gravity waves on deep water when damping and amplification effects are considered. The free surface elevation is written as follows:

$$
\eta(x, t)=\psi(x, t) \exp \left[\mathrm{i}\left(k_{0} x-\Omega_{0} t\right)\right]+\text { c.c. }+O\left(\epsilon^{2}\right),
$$

where c.c. denotes the complex conjugate. The parameter, $\epsilon$, is a small parameter $(\epsilon \ll 1)$ used to carry out the multiple scale analysis leading to (3.1). The elevation, $\eta$, and envelope, $\psi$, are of order $O(\epsilon)$. We have assumed that the fluid viscosity, $\nu$, and density ratio, $\rho_{\mathrm{a}} / \rho$, are small: $\nu / \sqrt{g / k^{3}}=\epsilon^{2}$ and $\rho_{\mathrm{a}} / \rho=\epsilon^{2}$. These assumptions are generally used for water and air/sea interface.

We rewrite (3.1) in a standard form by using the following transformations:

$$
\xi=2 k_{0}(x-V t), \quad \tau=\Omega_{0} t, \quad \Psi=\sqrt{2} k_{0} \psi,
$$

leading to the following perturbed NLS:

$$
{ }_{\imath} \Psi_{\tau}-\frac{1}{2} \Psi_{\xi \xi}-|\Psi|^{2} \Psi={ }_{\imath} K \Psi,
$$

with

$$
K=\frac{\mathscr{W} k_{0}}{2 g \rho}-2 \frac{\nu k_{0}^{2}}{\Omega_{0}} .
$$

The sign of $K$ determines the nature of the perturbation. If $K>0$ we have amplification of waves and if $K<0$ we have depletion. For $K<0$, this perturbed NLS equation is similar to the NLS equation considered by Segur et al. (2005a). It is also similar to the NLS equation used by Bridges \& Dias (2007) when their coefficients $a$ and $c$ vanish. In that case the latter authors demonstrated that there is no enhancement of the modulational instability, whereas when $a>0$ the instability is enhanced.

The condition $K>0$ implies that

$$
\frac{4 v \kappa^{2} \Omega_{0}}{\beta s u_{*}^{2}}<1
$$

For a given friction velocity, this condition states that only carrier wave with frequency or wavenumber less than a threshold value may suffer modulational instability. Within the framework of the NLS equation, we consider weakly modulated wave train. Hence, we can assume that $\beta$ depends on the frequency (or wavenumber) and phase velocity 


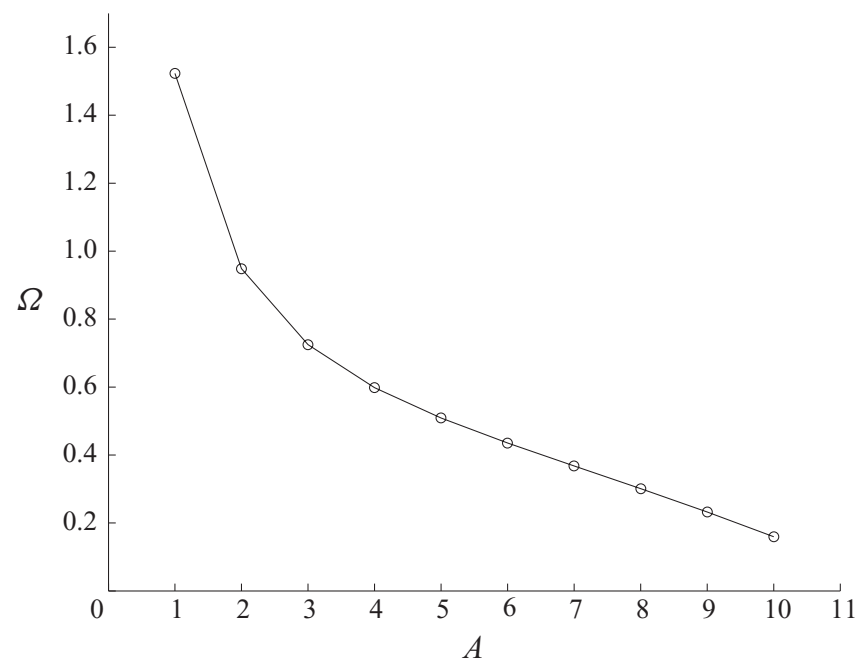

FIGURE 1. Normalized critical frequency $\Omega=\Omega^{c} /\left(s g^{2} / 4 v\right)^{1 / 3}$ as a function of $A=\kappa c_{0} / u_{*}$ (proportional to wave age) for $\kappa^{2} g z_{0} / u_{*}^{2}=3 \times 10^{-3}$.

of the carrier wave. Note that the computation of the coefficient $\beta$ is done through the numerical resolution of the Rayleigh equation. Conte \& Miles (1959) computed the values of $\beta$ as a function of $\kappa c_{0} / u_{*}$ for a logarithmic wind profile of the form

$$
U(z)=\frac{u_{*}}{\kappa} \ln \left(z / z_{0}\right),
$$

where $z_{0}$ is the roughness length given by $z_{0}=\alpha_{c h} u_{*}^{2} / g\left(\alpha_{c h}\right.$ is the Charnock constant $\approx 0.011-0.018$ ). Note that $c_{0}=\Omega_{0} / k_{0}$.

Introducing the dimensionless term $\kappa c_{0} / u_{*}$ proportional to the wave age $c_{0} / u_{*},(3.6)$ may be rewritten as follows:

$$
\Omega_{0}<\left[\frac{s g^{2} \beta}{4 v\left(\kappa c_{0} / u_{*}\right)^{2}}\right]^{1 / 3} .
$$

Let $\Omega^{c}$ be defined as follows:

$$
\Omega^{c}=\left(\frac{s g^{2}}{4 v}\right)^{1 / 3}\left(\frac{\beta}{\left(\kappa c_{0} / u_{*}\right)^{2}}\right)^{1 / 3} .
$$

Hence, (3.8) becomes

$$
\Omega_{0}<\Omega^{c} \text {. }
$$

Using the results of Conte \& Miles (1959, table 1), the values of $\Omega^{c} /\left(s g^{2} / 4 v\right)^{1 / 3}$ are plotted in figure 1 as a function of $\kappa c_{0} / u_{*}$ for the dimensionless roughness length $\kappa^{2} g z_{0} / u_{*}^{2}=3 \times 10^{-3}$. This value of the dimensionless roughness length corresponds to an aerodynamically smooth flow. The solid line divides the plane into modulationally unstable region and modulationally stable region. Wavetrains for which the point $\left(\kappa c_{0} / u_{*}, \Omega_{0} /\left(s g^{2} / 4 v\right)^{1 / 3}\right)$ belongs to the lower region suffer modulational instability. Otherwise dissipation prevents instability developing over in time. For fixed $\Omega_{0}$, instability prevails when $u_{*}$ fulfils the following relation:

$$
u_{*}>u_{*}^{c},
$$




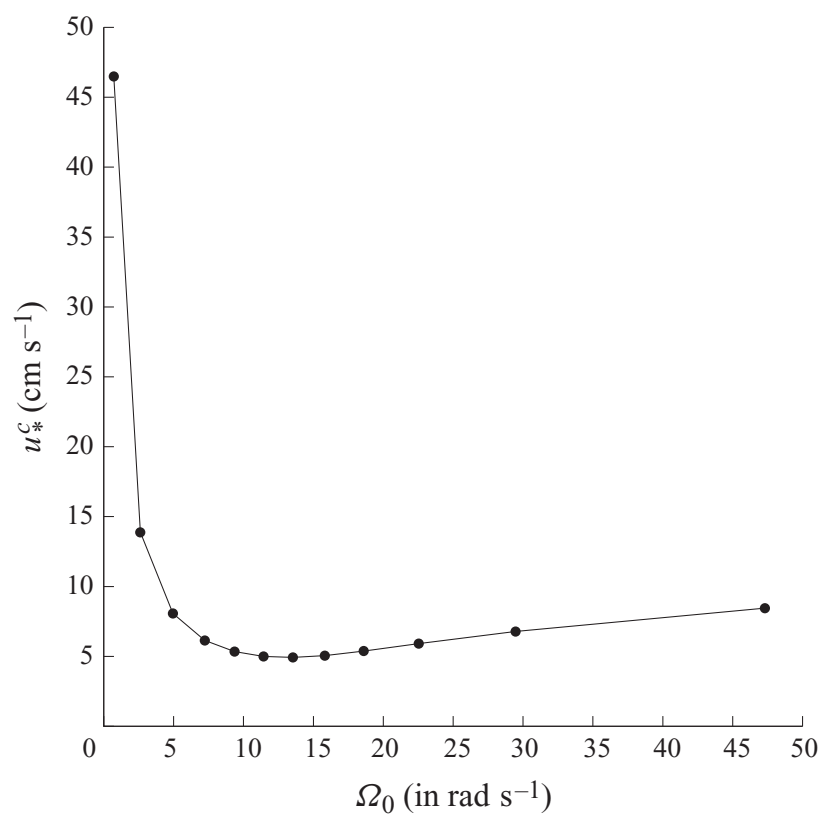

FIGURE 2. Critical friction velocity $u_{*}^{c}\left(\right.$ in $\mathrm{cm} \mathrm{s}^{-1}$ ) as a function of the carrier wave frequency $\Omega_{0}\left(\right.$ in $\left.\operatorname{rad~s}^{-1}\right)$ for $\kappa^{2} g z_{0} / u_{*}^{2}=3 \times 10^{-3}$.

where $u_{*}^{c}$ satisfies the equation

$$
\left[\frac{\beta^{c}}{\left(\kappa g / \Omega_{0} u_{*}^{c}\right)^{2}}\right]^{1 / 3}=\frac{\Omega_{0}}{\left(s g^{2} / 4 v\right)^{1 / 3}} .
$$

We recall that $\beta$ depends on $\kappa c_{0} / u_{*}$ or $\kappa g / \Omega_{0} u_{*}^{c}$ implicitly. Hence, $\beta^{c}$ is the value taken by $\beta$ when $\kappa g / \Omega_{0} u_{*}=\kappa g / \Omega_{0} u_{*}^{c}$. The quantity $u_{*}^{c}$ defines the minimum friction velocity induced by the wind to amplify a wave train whose carrier frequency is $\Omega_{0}$. For instance, for a carrier wave frequency $\Omega_{0}=4.886 \mathrm{rad} \mathrm{s}^{-1}$, the minimum friction velocity is $u_{*}^{c}=8 \mathrm{~cm} \mathrm{~s}^{-1}$, whereas for $\Omega_{0}=0.706 \mathrm{rad} \mathrm{s}^{-1}$ we found $u_{*}^{c}=$ $46 \mathrm{~cm} \mathrm{~s}^{-1}$. These computations correspond to $\kappa^{2} g z_{0} / u_{*}^{2}=3 \times 10^{-3}, v=10^{-2} \mathrm{~cm}^{2} \mathrm{~s}^{-1}$, $g=980 \mathrm{~cm} \mathrm{~s}^{-2}$ and $s=1.2 \times 10^{-3}$. For $\kappa^{2} g z_{0} / u_{*}^{2}=10^{-2}$ and $\Omega_{0}=1.73 \mathrm{rad} \mathrm{s}^{-1}$, the threshold value of the friction velocity is $u_{*}^{c}=22.6 \mathrm{~cm} \mathrm{~s}^{-1}$. The values of the critical friction velocity, $u_{*}^{c}$, as a function of the carrier wave frequency, $\Omega_{0}$, are plotted in figure 2 for $\kappa^{2} g z_{0} / u_{*}^{2}=3 \times 10^{-3}$. In the low-frequency regime stronger wind velocities are needed to sustain the modulational instability than for high-frequency water waves. For a given carrier frequency, a larger friction velocity is needed to sustain modulational instability when the roughness length is increased as shown in figure 3 .

To justify the use of the Miles' mechanism, we followed Janssen (2004) who showed that this mechanism seems to provide an adequate model for $\kappa c_{0} / u_{*}>4$. Furthermore, he emphasized that the inviscid Miles' model gives good agreement for slow waves too $\left(\kappa c_{0} / u_{*}<4\right)$, regarding the growth rate of waves by wind, with in situ data (see Janssen 2004, figure 3.3).

\section{The Stokes-like wave and its stability}

We call a solution of (3.4) a Stokes-like wave if it does not depend on $\xi$. In our case, the solution is 


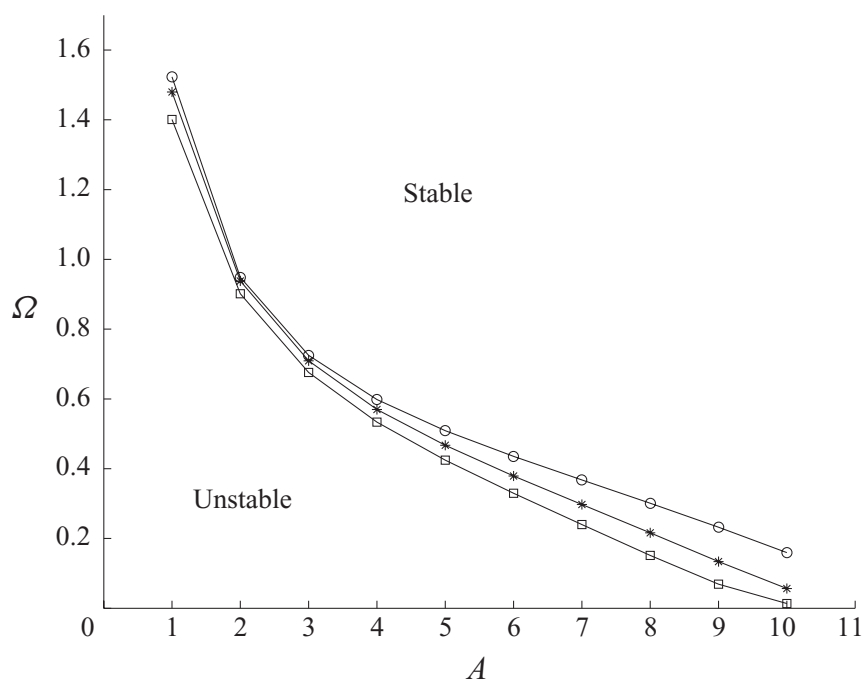

FIGURE 3. Normalized critical frequency $\Omega=\Omega^{c} /\left(s g^{2} / 4 v\right)^{1 / 3}$ as a function of $A=\kappa c_{0} / u_{*}$ (proportional to wave age) for several values of the roughness length: $\kappa^{2} g z_{0} / u_{*}^{2}=3 \times 10^{-3}$ (circle), $\kappa^{2} g z_{0} / u_{*}^{2}=1 \times 10^{-2}\left(\right.$ star) and $\kappa^{2} g z_{0} / u_{*}^{2}=2 \times 10^{-2}$ (square).

$$
\Psi_{s}=a(\tau) \mathrm{e}^{-l b(\tau)},
$$

with

$$
\begin{gathered}
a(\tau)=\tilde{a} \mathrm{e}^{K \tau}, \\
b(\tau)=\frac{\tilde{a}^{2}}{2 K} \mathrm{e}^{2 K \tau}+\Delta,
\end{gathered}
$$

where $\tilde{a}$ is a constant and $\Delta$ is put equal to $-\left(\tilde{a}^{2} / 2 K\right)$. To study the modulational stability of this solution we follow Segur et al. (2005a) and Leblanc (2007), and we superimpose to the solution (4.1) a small perturbation

$$
\Psi=\Psi_{s}(1+\delta \zeta(\xi, \tau))
$$

where $\delta \ll 1$. We then substitute (4.4) into (3.4), keeping only terms up to order $\delta$. We separate the real and imaginary parts of $\zeta(\xi, \tau)$ as $\zeta=M(\xi, \tau)+\imath N(\xi, \tau)$. The resulting equations are

$$
\begin{gathered}
M_{\tau}-\frac{1}{2} N_{\xi \xi}=0, \\
N_{\tau}+\frac{1}{2} M_{\xi \xi}+2 a^{2} M=0 .
\end{gathered}
$$

Now setting $M(\xi, \tau)=\operatorname{Re}\left(M_{0}(\tau) \mathrm{e}^{i l \xi}\right)$ and $N(\xi, \tau)=\operatorname{Re}\left(N_{0}(\tau) \mathrm{e}^{l \xi \xi}\right), \quad l$ being the wavenumber of the perturbation, we arrive at the following system of equations:

$$
\begin{aligned}
& \frac{\mathrm{d} M_{0}}{\mathrm{~d} \tau}+\frac{1}{2} l^{2} N_{0}=0, \\
& \frac{\mathrm{d} N_{0}}{\mathrm{~d} \tau}+\left(2 a^{2}-\frac{1}{2} l^{2}\right) M_{0}=0 \quad \text { with } \quad a^{2}=\tilde{a}^{2} \mathrm{e}^{2 K \tau} .
\end{aligned}
$$

Equations (4.7) and (4.7) can be combined to give

$$
\frac{\mathrm{d}^{2} M_{0}}{\mathrm{~d} \tau^{2}}+\frac{1}{2} l^{2}\left[\frac{1}{2} l^{2}-2 a^{2}\right] M_{0}=0 .
$$


Let $\tau=2 r / l^{2}$ and $\lambda=2 \tilde{a} / l$. Then (4.9) becomes

$$
\frac{\mathrm{d}^{2} M_{0}}{\mathrm{~d} r^{2}}+\left(1-\lambda^{2} \exp \left(4 K r / l^{2}\right)\right) M_{0}=0
$$

One may distinguish the following two cases according to whether $K$ is negative or positive.

(i) Let $K$ be negative. This case was analysed by Segur et al. (2005a), and it was demonstrated that the modulational instability is restabilized by dissipation. To summarize, it was shown in the presence of damping that the linear perturbation can grow, but its growth is limited whereas in the absence of damping the perturbation grows without bound. According to our previous linear stability analysis, instability restabilizes after a period of time of order equal to $\ln (2 \tilde{a} / l) /|K|$ (or $\tau$ is $O(\ln (2 \tilde{a} / l)$ $\tau_{0} / 2 \pi|K|$, where $\tau_{0}$ is the period of the carrier wave). This value corresponds to the change of sign of the coefficient of $M_{0}$ in (4.9). Note that Segur et al. (2005a) extended their linear stability analysis to the nonlinear case, too. This case is illustrated in figure 4 from numerical simulations of the NLS equation (3.4) which show the temporal evolution of the normalized amplitude of the carrier wave and most unstable sidebands $(l=0.20)$ with dissipation and forcing $(K=-0.0004)$ and without dissipation and forcing $(K=0)$. The amplitudes are normalized by the initial amplitude of the carrier wave namely $\tilde{a}$. The solid and dashed lines correspond to the amplitude of the carrier and sidebands, respectively. At $\tau=0$, the initial wave steepness of the Stokes wave is 0.07 , and the ratio between the amplitudes of the sidebands and carrier wave is 0.10 . Furthermore, the carrier of the unperturbed Stokes wave given by (4.2) has been plotted in figure 4 . When $K$ is negative the perturbed solution is close to the unperturbed solution for large times, whereas when $K$ is positive the deviation between the two solutions increases with time. The case $K<0$ illustrated in figure 4 corresponds to the simulation, where initially the nonlinear term of the NLS equation is much more larger than the dissipative term $\left(\tilde{a}^{2} \gg|K|\right)$. This situation was discussed by Segur et al. (2005a, see their comment (iii) p. 238), and it was claimed that even with substantial growth of the perturbation, the Stokes solution of (3.4) is still linearly stable: it is always possible to find a gap (denoted $\Delta$ ) between unperturbed and perturbed solution that satisfies the linear stability criterion. In real oceanic situations, the dissipation due to viscosity (when ignoring breaking waves) is very weak compared to the nonlinear interactions. Hence, we can expect that small dissipation may not have time to play a role in the initial dynamics as it is demonstrated in figure 4. For large and negative values of $K$ the exponential function will rapidly become negligible and the solutions of (4.10) will converge asymptotically to the solutions of the equation $\mathrm{d}^{2} M_{0} / \mathrm{d} r^{2}+M_{0}=0$ which are oscillatory. There is no amplification of the modulational perturbation due to damping effect which is the dominant mechanism.

(ii) Let $K$ be positive. From our linear stability analysis, the modulational perturbations grow exponentially with time when $\left(l^{2} / 2-2 \tilde{a}^{2} \mathrm{e}^{2 K \tau}\right)<C$, where $C$ is a strictly negative constant. This behaviour is also observed within the framework of the NLS equation as shown in figure 4 (bottom) from the numerical simulation of (3.4) for the most unstable perturbation $(l=0.2)$. In this case $K=0.0004$ and the initial wave steepness of the Stokes wave is 0.07 . When $\left(l^{2} / 2-2 \tilde{a}^{2} \mathrm{e}^{2 K \tau}\right)>0$ the perturbations are oscillatory for $\tau<\ln (l / 2 \tilde{a}) / K\left(\tau<\ln (2 \tilde{a} / l) \tau_{0} / 2 \pi|K|\right)$ and grow exponentially for $\tau>\ln (l / 2 \tilde{a}) / K\left(\tau>\ln (2 \tilde{a} / l) \tau_{0} / 2 \pi|K|\right)$. This behaviour holds in the nonlinear case, too. Figure 5 shows the destabilization of an initially stable perturbation $(l=0.40)$ due to forcing within the framework of the numerical simulation of NLS 

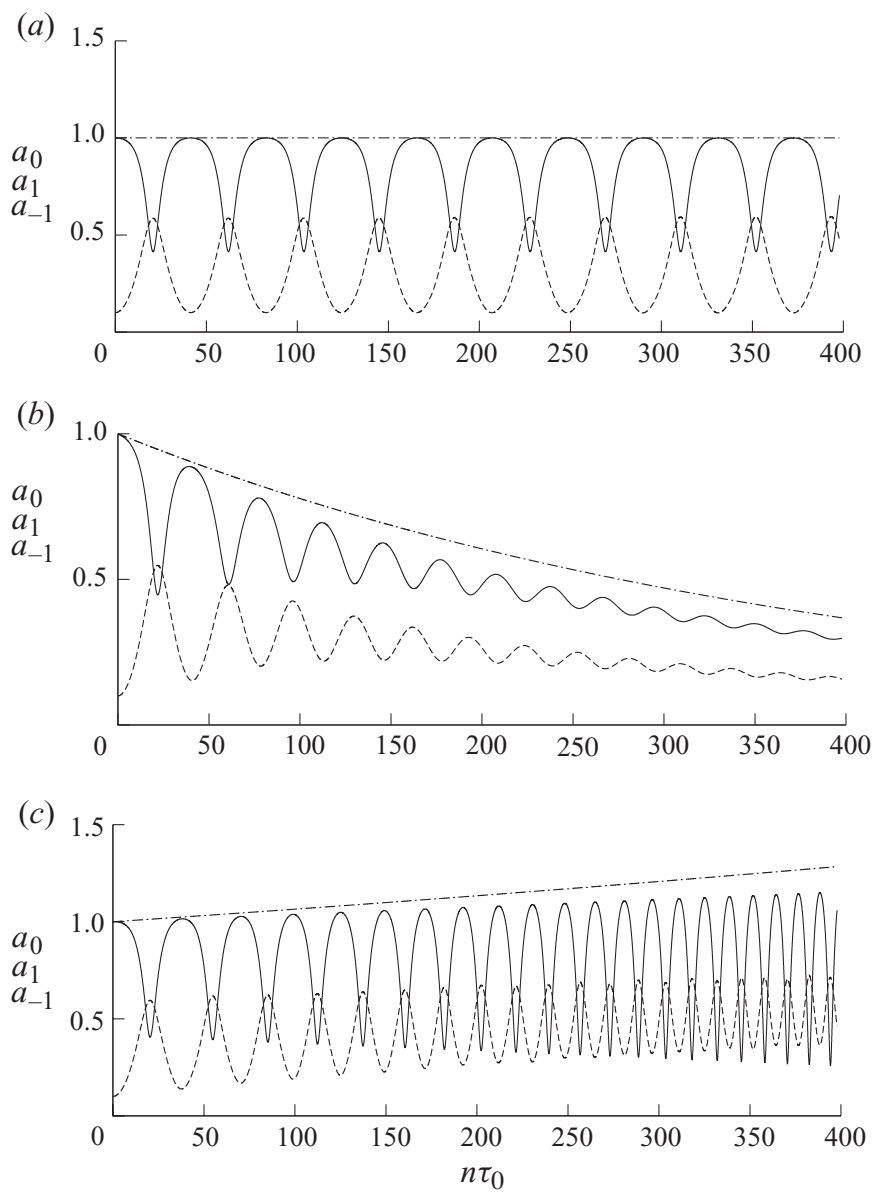

FiguRE 4. Temporal evolution of the normalized amplitude of the carrier wave, $a_{0}$, (solid line) and upper and lower sidebands, $a_{1}$ and $a_{-1}$ (dashed line) corresponding to the most unstable perturbation $(l=0.20)$ without dissipation and wind $(a)$, in the presence of dissipation and wind for $K=-0.0004(b)$ and for $K=0.0004(c)$ within the framework of numerical simulations of (3.4). The dash-dotted line corresponds to the carrier of the unperturbed Stokes wave given by (4.2). The period of the carrier wave is $\tau_{0}$.

equation (3.4). During the first stage, the perturbation is oscillatory and then grows exponentially. Beyond $\tau=150 \tau_{0}$, the gap between the unperturbed and perturbed solutions increases with time. In the wavenumber space, the region of instability increases as time increases. Therefore, stable modes become unstable after some time. In this simulation, the initial wave steepness of the wave train is 0.07 and the ratio between the amplitudes of the sidebands and carrier wave is 0.10 . For large and positive values of $K$, the coefficient $1-\lambda^{2} \exp \left(4 K r / l^{2}\right)$ will become rapidly negative. Hence, $\mathrm{d}^{2} M_{0} / \mathrm{d} r^{2}$ will have the sign of $M_{0}$ and the solution of (4.10) corresponds to the temporal amplification of the modulational perturbation of the basic solution given by (4.1). In this case the wind effect dominates.

For small values of $|K|$, the exponential function varies slowly and for small values of $r$ the coefficient $1-\lambda^{2} \exp \left(4 K r / l^{2}\right)$ is close to $1-\lambda^{2}$. Hence, when $\lambda<1$ or $l>2 \tilde{a}$ the solutions remain bounded (modulational perturbations are not amplified) and when $\lambda>1$ or $l<2 \tilde{a}$ the solutions diverge (modulational perturbations are amplified). 


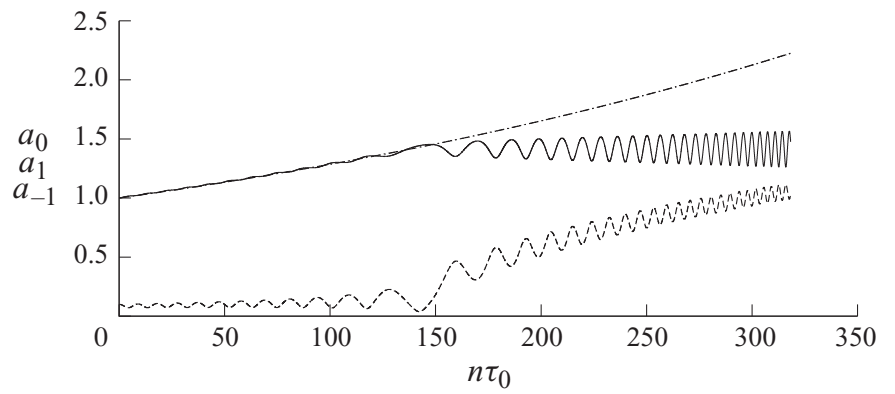

FIGURE 5. Temporal evolution of the normalized amplitude of the carrier wave, $a_{0}$, (solid line) and upper and lower sidebands, $a_{1}$ and $a_{-1}$ (dashed line) corresponding to a stable perturbation $(l=0.40)$ in the presence of dissipation and wind $(K=0.0001)$ within the framework of numerical simulations of (3.4). The dash-dotted line corresponds to the carrier of the unperturbed Stokes wave given by (4.2). The period of the carrier wave is $\tau_{0}$.

Consequently, for $K<0$ any modulational perturbation is stable or becomes stable after some time (as shown by Segur et al. 2005a), whereas for $K>0$ any modulational perturbation is unstable or becomes unstable after some time. For values of $K$ close to zero, which corresponds to a quasi-equilibrium between wind and damping effects, we can conclude that modulational perturbations of wavenumber $l$ will be linearly unstable when $l<2 \tilde{a}$ and linearly stable otherwise (classical result).

\section{Discussion}

We have shown that the joint action of wind and dissipation have additive effects on the modulation of a wave train. It has been argued and experimentally demonstrated that the effect of dissipation on a wave train is to stabilize the Benjamin-Feir instability (Segur et al. 2005a). In the regime we are considering, we have shown that the effect of the wind is contrary, as expected (Leblanc 2007). However, both effects, although additive, do not have the same dependence on the frequency (or wavenumber) of the carrier wave $\left(\Omega_{0}\right)$. Consequently, the existence or not of the Benjamin-Feir instability under the action of wind and taking dissipation into account depends on the strength of the friction velocity at the water surface. We found that the critical wind velocity, $u_{*}^{c}$, increases with the wavelength of the carrier wave. In the presence of wind and dissipation, the unstable domain shrinks for low-frequency regime: this means that young waves are more sensitive to modulational instability than old waves.

In this paper, we have considered a weakly nonlinear model (the NLS equation) and a linear wind-wave coupling. The next step is to consider the fully nonlinear water wave equations with linear and nonlinear wind-wave modelling.

We thank the anonymous referees for their helpful comments. R. A. K. thanks IRPHE (Marseille, France) for kind hospitality and CNPq (Brazil) for partial financial support.

\section{REFERENCES}

Alber, I. E. 1978 Proc. R. Soc. Lond. A 363, 525-546.

Alber, I. E. \& Saffman, P. G. 1978 TWR Defense and Space Systems Group Rep. 31326-6035-RU-00. Benjamin, T. B. \& Feir, J. E. 1967 J. Fluid Mech. 27, 417-430.

Bliven, L. F., Huang, N. E. \& Long, S. R. 1986 J. Fluid Mech. 162, 237-260. 
Modulational instability in deep water under the action of wind and dissipation 149

Bridges, T. J. \& Dias, F. 2007 Phys. Fluids 19, 101063.

Conte, S. D. \& Miles, J. W. 1959 J. Soc. Ind. Appl. Maths 7, 361-366.

Crawford, D. R., Saffman, P. G. \& Yuen, H. C. 1980 Wave Motion 2, 1-16.

Dias, F., Dyachenko, A. I. \& Zakharov, V. E. 2008 Phys. Lett. A 371, 12971302.

Janssen, P. A. E. M. 2004 The Interaction of Ocean Waves and Wind. Cambridge University Press.

LaMB, H. 1993 Hydrodynamics. Dover.

Leblanc, S. 2007 Phys. Fluids 19, 101705.

Lighthill, M. J. 1965 J. Inst. Maths Appl. 1, 269-306.

Lundgren, T. S. 1989 In SIAM Proceedings (ed. R. E. Caflisch), ISBN 0-89871-235-1.

Miles, J. W. 1957 J. Fluid Mech. 3, 185-204.

Miles, J. W. 1967 Proc. R. Soc. Lond. A 297, 459-475.

MiLes, J. W. 1996 J. Fluid Mech. 322, 131-145.

Segur, H., Henderson, D., Carter, J., Hammack, J., Li, C. M., Pheiff, D. \& Socha, K. $2005 a$ J. Fluid Mech. 539, 229-271.

Segur, H., Henderson, D. M. \& Hammack, J. L. 2005 b In Proceedings of the 14th 'Aha Huliko' a Hawaiian Winter Worshop, pp. 43-57.

Skandrani, C., Kharif, C. \& Poitevin, J. 1996 Contemp. Maths 200, 157-171.

Stokes, G. G. 1847 Camb. Phil. Soc. Trans. 8, 441-455.

Waseda, T. \& Tulin, M. P. 1999 J. Fluid Mech. 401, 55-84.

Whitham, G. B. 1974 Linear and Nonlinear Waves. Wiley Interscience.

Wu, G., Liu, Y. \& Yue, D. K. P. 2006 J. Fluid Mech. 556, 45-54.

Zakharov, V. E. 1968 J. Appl. Mech. Tech. Phys. 9, 190-194. 